PROCEEDINGS OF THE

AMERICAN MATHEMATICAL SOCIETY

Volume 134, Number 3, Pages 697-701

S 0002-9939(05)08412-1

Article electronically published on October 17, 2005

\title{
A NOTE ON A SYMMETRY RESULT FOR TRAVELING WAVES IN CYLINDERS
}

\author{
C. E. KENIG AND F. MERLE
}

(Communicated by David S. Tartakoff)

\begin{abstract}
We prove in this note that all bounded traveling waves, in cylinders, of some $N$-dimensional viscous conservation laws are symmetric.
\end{abstract}

\section{THE MAIN RESULT}

In this note, we consider traveling wave solutions of the equation

$$
\frac{\partial U}{\partial t}=\triangle U-\sum_{i=1}^{N} \frac{\partial}{\partial_{x_{i}}}\left(f_{i}(U)\right)
$$

for $\left(x_{1}, x^{\prime}\right) \in \mathbb{R} \times T^{N-1}$ and $f_{i} \in C^{2}(\mathbb{R}, \mathbb{R})$. That is, we consider solutions of the form

where $u$ satisfies

$$
U(t, x)=u\left(x_{1}-c t, x^{\prime}\right),
$$

$$
\triangle u-\left(f_{1}(u)-c u\right)_{x_{1}}-\sum_{i=2}^{N}\left(f_{i}(u)\right)_{x_{i}}=0
$$

for $\left(x_{1}, x^{\prime}\right) \in \mathbb{R} \times T^{N-1}$.

The main result of this note is the following.

Theorem 1. Assume that $u$ is an $L^{\infty}$ solution of (1.2).

i) Then there are $a_{ \pm}$such that $\forall x^{\prime} \in T^{N-1}, \lim _{x_{1} \rightarrow \pm \infty} u,\left(x_{1}, x^{\prime}\right)$ is defined and equal to $a_{ \pm}$. In addition, the limit is uniform in $x^{\prime} \in T^{N-1}$.

ii) Assume in addition that

$$
f_{1}^{\prime}\left(a_{ \pm}\right)-c \neq 0 .
$$

Then $u\left(x_{1}, x^{\prime}\right)=v\left(x_{1}\right)$, where

$$
v_{v_{1} x_{1}}-\left(f_{1}(v)-c v\right)_{x_{1}}=0 \quad \text { for } x_{1} \in \mathbb{R} .
$$

Remark. If $f_{i} \equiv 0$ for $i \geq 2$, or in some sense the degeneracy of $f_{i}$ at $a_{ \pm}$is of higher order than the one of $f_{1}$, the degeneracy condition in part (ii) of the theorem can be relaxed.

Received by the editors April 24, 2004.

2000 Mathematics Subject Classification. Primary 35B40, 35B99, 35L65.

The first author was partially supported by the NSF, and at IAS by the von Neumann Fund, the Weyl Fund, the Oswald Veblen Fund and the Bell Companies Fellowship.

(C)2005 American Mathematical Society Reverts to public domain 28 years from publication 
Under the assumptions of Theorem 1, we have the following.

Theorem 2 (Liouville Theorem for (1.1)). Let $u(x)$ be a solution of equation (1.2). Consider $U(t, x)$ to be a solution of equation (1.1) defined for all time $t \in \mathbb{R}$ such that for a constant $C_{0}>0$,

$$
\forall t \in \mathbb{R}, \quad\left\|U(t, x)-u\left(x_{1}-c t\right)\right\|_{L^{1}} \leq C_{0} .
$$

Then there is an $x_{0} \in \mathbb{R}$ such that

$$
\forall t \in \mathbb{R}, \forall x \in \mathbb{R} \times T^{N-1}, \quad U(t, x)=u\left(x_{1}-c t+x_{0}\right) .
$$

From this result, we can then derive the following asymptotic stability result for equation (1.1).

Theorem 3 (Asymptotic Stability for Traveling Waves). Let $U(t, x)$ be a solution of equation (1.1) for $t>0, x \in \mathbb{R} \times T^{N-1}$ and initial data

$$
U(0, x)=U_{0}(x) .
$$

Assume in addition that for $A>0$ and for a travelling wave $u$,

$$
\left\|U_{0}\left(x_{1}, x^{\prime}\right)-u\left(x_{1}\right)\right\|_{L^{1}\left(\mathbb{R} \times T^{N-1}\right)} \leq A .
$$

Then there exists a function $g_{A}(t)$ depending only on $A, u$ with $g_{A}(t) \rightarrow 0$ as $t \rightarrow+\infty$, such that, for all such $u$, we have

$$
\forall t>1, \operatorname{Inf}_{x_{10} \in \mathbb{R}}\left\|U(t, .)-u\left(.+x_{10}-c t\right)\right\|_{L^{\infty}\left(\mathbb{R} \times T^{N-1}\right)} \leq g_{A}(t) .
$$

The proofs of Theorems 2, 3 are completely similar to the corresponding ones of [1] as soon as Theorem [1 is proved. We therefore devote the rest of this paper to the proof of Theorem 1 .

\section{Proof of Theorem 1}

Let $u$ be an $L^{\infty}$ solution of (1.2) and let $C=\mathbb{R} \times T^{n-1}$. We first prove the following.

Lemma 1. We have

$$
\begin{aligned}
\lim _{x_{1} \rightarrow+\infty} u\left(x_{1}, x^{\prime}\right) & =a_{+}, \\
\lim _{x_{1} \rightarrow-\infty} u\left(x_{1}, x^{\prime}\right) & =a_{-},
\end{aligned}
$$

uniformly in $x^{\prime} \in T^{N-1}$, where $\left(a_{+}, a_{-}\right)$are $\left(\operatorname{Sup}_{C} u, \operatorname{In} f_{C} u\right)$ or $\left(I n f_{C} u, S u p_{C} u\right)$.

Proof. We first remark from standard elliptic theory that

$$
u \in C^{3}\left(\mathbb{R} \times T^{N-1}, \mathbb{R}\right) \text { and }|u|_{C^{3}} \leq M .
$$

Moreover, if $u$ achieves a local maximum or a local minimum, from the strong maximum principle we have

$$
u\left(x_{1}, x^{\prime}\right) \equiv \text { constant } \equiv a,
$$

and all the conclusions of Theorem 1 hold. We now assume that $u\left(x_{1}, x^{\prime}\right)$ is different from a constant solution. 
From the fact that there are no local extrema, $\operatorname{In} f_{C}$ and $S u p_{C}$ are not achieved and there are $\left(y_{1 n}, y_{n}^{\prime}\right) \in \mathbb{R} \times T^{N-1}$ (respectively $\left.\left(z_{1 n}, z_{n}^{\prime}\right) \in \mathbb{R} \times T^{N-1}\right)$ such that

$$
\begin{aligned}
& u\left(y_{1 n}, y_{n}^{\prime}\right) \underset{n \rightarrow+\infty}{\longrightarrow} \operatorname{Sup}_{C} u=a_{+}, \\
& u\left(z_{1 n}, z_{n}^{\prime}\right) \underset{n \rightarrow+\infty}{\longrightarrow} \text { Inf } f_{C} u=a_{-},
\end{aligned}
$$

with $\left|y_{1 n}\right| \rightarrow+\infty$ and $\left|z_{1 n}\right| \rightarrow+\infty$.

One can assume, eventually extracting a subsequence, that

$$
y_{1 n} \rightarrow+\infty \text { and } y_{1 n}<y_{1 n+1} .
$$

(The proof in the other case is identical.)

Let us now prove

$$
\operatorname{Inf} f_{x^{\prime} \in T^{N-1}} u\left(y_{1 n}, x^{\prime}\right) \underset{n \rightarrow+\infty}{\longrightarrow} a_{+} .
$$

Indeed, let us consider

$$
u_{n}\left(x_{1}, x^{\prime}\right)=u\left(y_{1 n}+x_{1}, x^{\prime}\right) .
$$

We have

- $\left|u_{n}\right|_{C^{3}} \leq C$,

- $u_{n}$ is solution of equation (1.2),

- $u_{n}\left(0, y_{n}^{\prime}\right) \underset{n \rightarrow+\infty}{\longrightarrow} a_{+}=\operatorname{Sup} u=\operatorname{Sup} u_{n}$.

Extracting a subsequence, we have for $W:\left(x_{1}, x^{\prime}\right) \mapsto W\left(x_{1}, x^{\prime}\right)$ and $y^{\prime} \in T^{N-1}$,

$$
u_{n}\left(x_{1}, x^{\prime}\right) \underset{C_{l o c}^{2}}{\longrightarrow} W\left(x_{1}, x^{\prime}\right) \text { and } y_{n}^{\prime} \rightarrow y^{\prime}
$$

where

$$
W \text { is solution of (1.2), SupW } \leq a_{+}, W\left(0, y^{\prime}\right)=a_{+} .
$$

Therefore,

$$
W \equiv a_{+}
$$

and since the result is true for all subsequences

$$
u\left(y_{1 n}+x_{1}, x^{\prime}\right) \underset{C_{l o c}^{2}}{\longrightarrow} a_{+},
$$

then (1.7) follows.

From the fact that $u$ does not have local minimum, we have

$$
a_{+} \geq \operatorname{Inf}_{\substack{x_{1} \in\left(y_{1 n}, y_{1(n+1)}\right) \\ x^{\prime} \in T^{N-1}}} u\left(x_{1}, x\right) \geq \operatorname{Inf}_{\substack{x_{1}=y_{1 n}, y_{1(n+1)} \\ x^{\prime} \in T^{N-1}}} u\left(x_{1}, x\right) \underset{n \rightarrow+\infty}{\longrightarrow} a_{+} .
$$

Since $y_{1 n}<y_{1(n+1)}$ and $y_{1 n} \longrightarrow_{n \rightarrow+\infty}+\infty$, then

$$
\lim _{x_{1} \rightarrow+\infty}\left\{\operatorname{Inf} f_{x^{\prime} \in T^{N-1}} u\left(x_{1}, x^{\prime}\right)\right\} \text { exists and equals } a_{+} \text {. }
$$

It follows that $z_{1 n} \rightarrow-\infty$ and by the same procedure,

$$
\lim _{x_{1} \rightarrow-\infty}\left\{\operatorname{Sup}_{x^{\prime} \in T^{N-1}} u\left(x_{1}, x^{\prime}\right)\right\} \text { exists and is equal to } a_{-} .
$$

This concludes the proofs of Lemma 1 and Theorem 1 part (i).

We now assume in addition a nondegeracy condition at $a_{+}, a_{-}$for $f_{1}(u)$, namely

$$
f_{1}^{\prime}\left(a_{+}\right)-c \neq 0 \text { and } f_{1}^{\prime}\left(a_{-}\right)-c \neq 0,
$$

and, for example, $a_{-}<a_{+}$. 
We first have the following estimates.

Lemma 2. There exist $\alpha>0$ and $C_{0}>0$ such that

$$
\begin{aligned}
& \left|u\left(x_{1}, x^{\prime}\right)-a_{+}\right| \leq C_{0} e^{-\alpha x_{1}}, \\
& \left|u\left(x_{1}, x^{\prime}\right)-a_{-}\right| \leq C_{0} e^{+\alpha x_{1}} .
\end{aligned}
$$

Proof. Let us prove the first one for example. The only question is when $x_{1} \rightarrow+\infty$.

(i) Let us first introduce

$$
w\left(x_{1}\right)=\frac{1}{\left|\operatorname{vol}\left(T^{N-1}\right)\right|} \int_{T^{N-1}}\left(a_{+}-u\left(x_{1}, x^{\prime}\right)\right) d x^{\prime} .
$$

We have

$$
w \geq 0 .
$$

Averaging the equation (1.2) over $T^{N-1}$ and using the periodic boundary conditions, we obtain that $w$ satisfies the following equation $\forall x_{1} \in \mathbb{R}$ :

$$
w_{x_{1} x_{1}}-\frac{\partial}{\partial x_{1}}\left[\frac{1}{\operatorname{vol}\left(T^{N-1}\right)} \int_{T^{N-1}}\left(f_{1}\left(u\left(x_{1}, x^{\prime}\right)\right)-c u\left(x_{1}, x^{\prime}\right)\right) d x^{\prime}\right]=0
$$

or equivalently

$$
w_{x_{1}}-\left[\frac{1}{\operatorname{vol}\left(T^{N-1}\right)} \int_{T^{N-1}}\left(f_{1}\left(u\left(x_{1}, x^{\prime}\right)\right)-c u\left(x_{1}, x^{\prime}\right)\right) d x^{\prime}\right] \equiv C_{0} .
$$

Define $\beta=f_{1}^{\prime}\left(a_{+}\right)-c$ and $\gamma=f_{1}\left(a_{+}\right)-c a_{+}$. We then have by linearization of the nonlinear term at $a_{+}$in (2.10):

$$
\forall x_{1} \in \mathbb{R}, \quad\left|w_{x_{1}}-\beta w-C_{0}+\gamma\right| \leq \operatorname{CSup}_{x^{\prime} \in T^{N-1}}\left|u\left(x_{1}, x^{\prime}\right)-a_{+}\right|^{2} .
$$

Since $w\left(x_{1}, x^{\prime}\right) \rightarrow 0$, as $x_{1} \rightarrow+\infty$, we have $C_{0}-\gamma=0$, and

$$
\forall x_{1} \in \mathbb{R},\left|w_{x_{1}}-\beta w\right| \leq C\left(\operatorname{Sup}_{x^{\prime} \in T^{N-1}}\left|u\left(x_{1}, x^{\prime}\right)-a_{+}\right|^{2}\right) .
$$

(ii) Relation between $u$ and $w$ : We now apply the Harnack principle as $x_{1} \rightarrow+\infty$ to $u$, and we get: there is a $C>0$ such that for $x_{1} \geq 0$,

$$
\operatorname{Sup}_{\substack{x^{\prime} \in T^{N-1} \\ \tilde{x}_{1} \in\left(x_{1}-1, x_{1}+1\right)}}\left(a_{+}-u\left(\tilde{x}_{1}, x^{\prime}\right)\right) \leq C \underset{\substack{x^{\prime} \in T^{N-1} \\ \tilde{x}_{1} \in\left(x_{1}-1, x_{1}+1\right)}}{\operatorname{Inf}}\left(a_{+}-u\left(\tilde{x}_{1}, x^{\prime}\right)\right),
$$

and, in particular, $\forall x_{1} \geq 0$,

$$
\operatorname{Sup}_{x^{\prime} \in T^{N-1}}\left(a_{+}-u\left(x_{1}, x^{\prime}\right)\right) \leq C w\left(x_{1}\right) .
$$

In particular, it is enough to prove the exponential decay for $w\left(x_{1}\right)$ to reach the conclusion of the lemma.

(iii) Exponential decay of $w$ : We have from (2.11) and (2.12),

$$
\forall x_{1} \geq 0, \quad\left|w_{x_{1}}-\beta w\right| \leq C w^{2} .
$$

Since $w \rightarrow 0$ as $x_{1} \rightarrow+\infty$ and $w>0$ we have $\beta<0$ and for $x_{1}$ large,

$$
-\frac{3}{2} \beta w \leq w_{x_{1}} \leq-\frac{\beta}{2} w
$$

which concludes the proof of Lemma 2, by integration in $x_{1}$.

We are now able to conclude the proof of Theorem 1 part (ii). We argue by contradiction: Assume there is $x_{1}^{0} \in \mathbb{R}$ such that for some $x_{0}^{\prime}, x_{1}^{\prime} \in T^{N-1}$,

$$
u\left(x_{1}^{0}, x_{0}^{\prime}\right) \neq u\left(x_{1}^{0}, x_{1}^{\prime}\right)
$$


Then using the periodicity, there are $x_{2}^{\prime}, x_{3}^{\prime} \in T^{N-1}$ such that

$$
u\left(x_{1}^{0}, x_{2}^{\prime}\right)=u\left(x_{1}^{0}, x_{3}^{\prime}\right)
$$

and

$$
\nabla_{x^{\prime}} u\left(x_{1}^{0}, x_{2}^{1}\right) \neq \nabla_{x^{\prime}} u\left(x_{1}^{0}, x_{3}^{\prime}\right) .
$$

Then

$$
H(t, x)=u\left(x_{1}-c t, x_{2}^{\prime}+x^{\prime}\right)-u\left(x_{1}-c t, x_{3}^{\prime}+x^{\prime}\right)
$$

is the difference of two solutions of equation (1.1) such that

i) $\forall t \in \mathbb{R}, \quad\|H(t)\|_{L^{1}(C)} \equiv$ constant,

ii) $H(0,(0,0))=0$ and $\nabla_{x^{\prime}} H(0,(0,0)) \neq 0$.

Applying Lemma 2.9 of [1], we obtain

which is a contradiction.

$$
\|H(1)\|_{L^{1}(C)}<\|H(0)\|_{L^{1}(C)}
$$

(Of course, multiplying $u\left(x_{1}, x_{2}+x^{\prime}\right)-u\left(x_{1}, x_{3}+x^{\prime}\right)$ by sign $\left[u\left(x_{1}, x_{2}+x^{\prime}\right)-\right.$ $\left.u\left(x_{1}, x_{3}+x^{\prime}\right)\right]$ yields a contradiction, by the same calculation as in the proof of Lemma 2.9 of [1.) This concludes the proof of Theorem 1.

\section{REFERENCES}

[1] C. Kenig and F. Merle. Asymptotic stability and Liouville theorem for scalar viscous conservation laws in cylinder, to appear in Comm. Pure Appl. Math.

Department of Mathematics, University of Chicago, Chicago, Illinois 60637-1514

- And - Institute for Advanced Study, Princeton, New Jersey 08540

Université de Cergy-Pontoise, Institut Universitaire de France, 33, Boulevard du Port, 95011 Cergy-Pontoise Cedex, France - and - Institute for Advanced Study, Princeton, New Jersey 08540 